\title{
Baldiges Aus
}

\section{für die FCKW-haltigen Dosieraerosole}

\author{
C. $A b t$
}

\begin{abstract}
Fluorchlorkohlenwasserstoff (FCKW) gelangt immer noch als Treibgas in zahlreichen Dosieraerosolen zur Behandlung der Atemwegserkrankungen zur Anwendung. Zum Schutz der Ozonschicht muss bis zum Jahr 2005 die Produktion und der Verbrauch von FCKW auch für medizinische Zwecke eingestellt werden. Mit welchen Konsequenzen müssen Ärztinnen und Ärzte sowie Patientinnen und $\mathrm{Pa}$ tienten rechnen?
\end{abstract}

\section{Problematik}

FCKW (Fluorchlorkohlenwasserstoff) ist ein altbewährtes Treibgas, welches zum Beispiel in Kühlschränken, Klimaanlagen und in diversen Sprühsprays seit 1930 weltweit zum Einsatz gelangt. Dank der inerten Eigenschaften wurde FCKW auch als Treibgas in Medikamentensprays eingesetzt. Seit 1956 steht eine Vielzahl von Wirkstoffen mittels FCKW-haltigen Dosieraerosolen für die Therapie von Atemwegserkrankungen zur Verfügung.

Die Dosieraerosole sind weltweit die am häufigsten verwendeten Wirkstoffapplikationssysteme in der Behandlung der Atemwegserkrankungen. Etwa 70 Millionen Patientinnen und Patienten in 100 Ländern benutzen ein Dosieraerosol. Insgesamt sollen weltweit 340 Millionen Dosieraerosole pro Jahr verwendet werden.

Wissenschaftler haben schon vor Jahren festgestellt, dass die Ozonschicht durch FCKW zerstört wird. Die Ozonschicht befindet sich in einer Höhe von 15-30 km über der Erdoberfläche in der sogenannten Stratosphäre und schützt uns vor einer direkten Ultraviolettbestrahlung. Vor allem die äusserst gefährliche Ultraviolett-B- und -C-Strahlung werden von der Ozonschicht absorbiert. Die Ozonschicht ist für das Leben auf der Erde überlebenswichtig. Ein Fehlen der Ozonschicht, wie das vor Millionen Jahren der Fall war, verunmöglicht das Leben auf der Erde. Eine zu-

Korrespondenz:

Dr. pharm. Carine Abt

Im Neusatz 2

CH-4144 Arlesheim nehmende UV-Strahlung begünstigt unter anderem die Entstehung von Hautkrebs, schwächt das Immunsystem und ist verantwortlich für diverse Augenerkrankungen. Auch wird das Wachstum von Pflanzen und kleinen Wasserorganismen behindert.

Die Dichte der Ozonschicht hat seit Beginn der siebziger Jahre um ungefähr 10\% abgenommen, was einen Anstieg der UV-Strahlung um rund 15\% verursacht hat. Das spektakulärste Zeichen dieser Zerstörung ist das Ozonloch. Mittlerweile hat das Ozonloch eine Rekordgrösse von 29,3 Millionen Quadratkilometern erreicht, was mehr als dreimal der Fläche von Europa entspricht.

Im Jahre 1987 unterschrieben 25 Staaten, darunter auch die Schweiz, das sogenannte «Protokoll von Montreal" über die die Ozonschicht zerstörenden Substanzen. Das Montreal-Abkommen, welches seither von über 150 Ländern unterzeichnet wurde, verbietet die Produktion und den Verbrauch von FCKW. Eine Produktion von FCKW ist vorläufig nur noch für die Herstellung von Dosieraerosolen im medizinischen Bereich erlaubt. FCKW wird ab 2005 voraussichtlich nicht mehr produziert und steht somit auch nicht mehr zur Verfügung.

Alle FCKW-Emissionen gefährden die Ozonschicht. Der Verbrauch von FCKW für medizinische Zwecke beträgt weltweit etwa 10000 Tonnen pro Jahr, was 0,5\% der ursprünglich produzierten FCKWMenge ausmacht. Auch die medizinischen Dosieraerosole werden deshalb auf ein alternatives, FCKWfreies Treibgas umgestellt oder durch eine andere Therapieform ersetzt werden müssen.

Zurzeit sind in der Schweiz schon mehrere Dosieraerosole auf ein FCKW-freies Treibgas umgestellt worden. Bei anderen Dosieraerosolen ist eine Umstellung geplant.

\section{Alternative Treibgase}

Das alternative Treibgas sollte nicht nur die gleichen Produkteigenschaften wie das FCKW (nicht toxisch, nicht reaktiv, nicht entzündbar, inert, geschmacksneutral usw.), sondern auch ähnliche physikalisch/ chemische Eigenschaften aufweisen. Die betroffenen Pharmafirmen haben sich in Form eines Konsortiums für die Erforschung und Entwicklung eines solchen Treibgases zusammengeschlossen.

Die heute oder demnächst in der Schweiz zur Verfügung stehenden alternativen Treibgase sind fluorierte Alkane, HFA-134a oder HFA 227.

Fluorierte Alkane haben zwar keine Auswirkungen auf die Ozonschicht, hingegen verbleiben sie während Jahrzehnten in der Atmosphäre und besitzen ein hohes Erwärmungspotential (das 1300- bzw. 3300 fache von $\mathrm{CO}_{2}$ ). Wegen des möglichen Treibhauseffektes stellen die fluorierten Treibgase nur eine Übergangslösung dar. 


\section{Therapeutische Möglichkeiten}

Die Inhalation ist derzeit ein unverzichtbarer Teil der Standardbehandlung von akuten und chronischen Atemwegserkrankungen. Das Medikament wird entweder in Form einer Inhalationslösung mit Hilfe eines Inhalationsapparates inhaliert oder die Wirksubstanzen werden mittels benutzerfreundlichen Dosieraerosolen oder Pulverinhalatoren eingeatmet. Das Medikament gelangt somit zielgerichtet direkt in die Bronchien und in die Lunge.

\section{Inhalationslösungen}

Die Inhalationslösung wird mit Hilfe eines Inhalationsapparates (z.B. Pari) inhaliert. Der Vorteil ist, dass die Inhalationslösung auf die individuellen $\mathrm{Pa}$ tientenbedürfnisse mittels Rezeptur konzipiert werden kann. Die Inhalationslösungen sind besonders geeignet für Säuglinge und Kleinkinder sowie für ältere Patienten und Patientinnen.

Gemäss Auskunft der Lungenliga Schweiz sind zurzeit über 28000 Inhalatoren in der Schweiz im Einsatz.

\section{Dosieraerosole}

Im Dosieraerosol liegt das Medikament entweder in gelöster oder in Form einer Suspension im flüssigen Treibgas vor. Bei der Auslösung des Sprühstosses wird das Medikament in feinste Tröpfchen oder Partikel im Treibgas vernebelt respektive zerstäubt und liegt so als Aerosol vor. Pro Sprühstoss wird eine bestimmte Menge des Medikamentes bzw. des Wirkstoffes freigesetzt.

Neu kommen in den Dosieraerosolen anstelle von FCKW fluorierte Treibgase zur Anwendung. Die Umformulierung der Dosieraerosole von FCKW auf fluorierte Alkane ist ein kompliziertes und kostenintensives Verfahren. Es müssen nicht nur die ideale Zusammensetzung der Wirk- und Hilfsstoffe gefunden, sondern zum Teil auch neue Sprayvorrichtungen entwickelt werden. Die klinischen Studien müssen für eine Registrierung teilweise wiederholt werden, um ein gleich günstiges Nutzen-Risiko-Verhältnis wie beim FCKW-haltigen Spray zu belegen. Damit ist aber nicht ausgeschlossen, dass je nach Präparat und Individuum bei der Umstellung auf ein FCKW-freies Dosieraerosol eine Dosisanpassung notwendig werden kann. In den nächsten Jahren wird versucht werden, alle Dosieraerosole nach und nach auf FCKWfreie Dosieraerosole umzustellen.

\section{Pulverinhalatoren}

Die Pharmaindustrie hat in den letzten 15 Jahren nicht nur intensiv nach neuen Substanzen gegen Atemwegserkrankungen, sondern auch nach neuen Inhalationstechnologien geforscht. Der grosse Innovationsschub, der sicherlich auch dank des drohenden Verbotes von FCKW-betriebenen Dosieraerosolen entstand, ergab eine Vielzahl von neuentwickelten Pulverinhalatoren wie z.B. Diskhaler, Turbuhaler, Diskus und Aerolizer.
Die Pulverinhalation wurde jedoch nicht nur aus Umweltgründen entwickelt, sondern auch, um den Wirkstoff besser in die Lunge zu befördern. Da das Pulver angesaugt werden muss, entfällt die problematische Koordination - wie beim Dosieraerosol von Sprayen und Einatmen.

Die Pulverinhalation hat sich vor allem in Ländern mit grossem Umweltbewusstsein durchgesetzt. In Schweden werden 90\% der Therapien in Form von Pulverinhalatoren appliziert. In der Schweiz werden zurzeit 40\% der Therapien als Pulverinhalatoren eingesetzt.

\section{Konsequenzen und Empfehlungen}

Bei Patienten/-innen mit Pulverinhalatoren wird sich überhaupt nichts ändern.

Aus Umweltschutz- wie auch aus medizinischen Gründen empfiehlt es sich, die Patienten/-innen möglichst auf Pulverinhalatoren ein- bzw. umzustellen.

Einzig für die folgenden Patientengruppen ist eine Pulverinhalation ungeeignet, da diese das Pulver nicht oder nur ungenügend an den Wirkort transportieren können:

- Patienten mit geringem Inspirationsfluss

(Flow: <30 Liter/Min.);

- Säuglinge und Kleinkinder bis 4 Jahre;

- Demente Patientinnen und Patienten;

- Notfälle oder intubierte Patientinnen und Patienten.

Für diese Patientengruppen bleibt die Möglichkeit einer Inhalationslösung oder eines Dosieraerosols vorzugsweise mit Vorschaltkammer.

Folgende Wirkstoffe sind derzeit als Präparate in FCKW-freier Form von der IKS zugelassen (Stand April 2001):

\section{Tabelle 1}

\begin{tabular}{|c|c|c|}
\hline Stoffklasse & Wirkstoff & $\begin{array}{l}\text { als FCKW-freie Form } \\
\text { erhältliche Präparate }\end{array}$ \\
\hline$\beta$-2-Stimulatoren & $\begin{array}{l}\text { Salbutamol } \\
\text { Fenoterol }\end{array}$ & $\begin{array}{l}\text { Airomir }{ }^{\circledR}, \text { Ventolin }^{\circledR} \\
\text { Berotec }{ }^{\circledR}\end{array}$ \\
\hline Glukokortikoide & $\begin{array}{l}\text { Beclomethason } \\
\text { Fluticason }\end{array}$ & $\begin{array}{l}\text { Aerobec }{ }^{\circledR *} \\
\text { Axotide }^{\circledR}\end{array}$ \\
\hline Kombinationen & $\begin{array}{l}\text { Salmeterol } \\
\text { und Fluticason }\end{array}$ & Seretide ${ }^{\circledR}$ \\
\hline
\end{tabular}

* Das Präparat oder dessen FCKW-freie Form wurde erst kürzlich zugelassen und ist derzeit noch nicht auf dem Markt.

Informieren Sie Ihre betroffenen Patienten/-innen über die bevorstehende Treibgasumstellung. Als Unterstützung stehen Ihnen die gratis zur Abgabe gestellten Patienteninformationsblätter zur Verfügung. (Bestelladresse siehe unter Hinweise.)

Stellen Sie bitte klar, dass die Umstellung für die Patientinnen und Patienten in der Regel kein Problem darstellt. Das Nutzen-Risiko-Verhältnis von FCKW- 
freien Dosieraerosolen ist gleich wie bei den bisherigen FCKW-haltigen Sprays, doch ist je nach Präparat und Individuum eine Dosisanpassung und damit vorübergehend eine engmaschigere Überwachung notwendig (vgl. Text der entsprechenden Fachinformation). Zudem sind leichte geschmackliche Veränderungen möglich.

\section{Hinweise}

Weitere Informationen zu den FCKW-freien Dosieraerosolen finden Sie unter:

- Website Lungenliga Schweiz (www.lung.ch),

- Website BUWAL (www.umwelt-schweiz.ch).

Wünschen Sie Patienteninformationsblätter (deutsch, französisch, italienisch), bestellen Sie bitte bei der EDMZ, 3003 Bern, unter Tel. 03132550 50, Fax 031 32550 58, E-mail: verkauf.zivil@bbl.admin.ch. 